\title{
The Relationship between Perceived Social Support and Social in College Students: The Mediation of Sense of Coherence
}

\author{
Jiao Jiang and Danna Luo* \\ Department of Education, Yunnan Normal University, 650500, Kunming, China
}

\begin{abstract}
This study aims to explore the relationship between the basic characteristics of college students' social anxiety and the perceived social support and sense of coherence, and further explore the mediating role of the sense of coherence between perceived social support and social anxiety. A questionnaire survey of 420 college students was conducted using the Interaction Anxiousness Scale (IAS), the Perceived Social Support Scale (PSSS) and the Sense of Coherence-13(SOC-13). The results showed that: (1) The social anxiety in contemporary college students was very high, and the phenomenon of social anxiety of female is significantly higher than male. (2) College students' social anxiety were significantly negatively correlated with both perceived social support and sense of coherence, and perceived social support was significantly related to sense of coherence. (3) Sense of coherence plays a partial mediation between perceived social support and social anxiety. This study provides theoretical support for revealing the mechanism between perceived social support and social anxiety.
\end{abstract}

\section{Introduction}

Social anxiety refers to the emotional experience and avoidance behavior of tension and fear when individual in social situation [1]. Social anxiety first appears in childhood and peaks in adolescence, commons among college students, and affecting their mental health, academic performance and normal interpersonal relationship [2-4]. And without intervention, it may develop social disorder [5]. In addition, a global prevalence of social anxiety was showed that $36 \%$ of young people met the threshold criteria for social anxiety disorder [6] therefore, it is necessary to explore the influencing factors of social anxiety in to prevent the occurrence of social anxiety in college students or to relieve social anxiety that has been produced.

According to social causation model, the lack of perceived social support led to social anxiety. Perceived social support refers to the social support experienced by individuals subjectively, which is closely related to the individual subjective feelings [7]. A large number of studies have shown that perceived social support is related to the level of

* Corresponding author: donna101512@163.com 
individual mental health, and individuals with perceived social support are less likely to with negative emotions, such as depression and anxiety [8, 9]. In studies of children and adolescents, perceived social support can reduce social anxiety. Individuals with fewer socially anxious perceiving themselves as having more social resources available, perceived social support produces a positive buffer effect on external pressure and subjective negative feelings [10-12]. So, the higher perceived social support is, the lower the level of social anxiety is.

Sense of coherence was first proposed by Antonovsky. It is a stable psychological tendency that comprehensively reflects the individual's pressure on the internal and external environment, the resource support in the face of pressure, and the perception of the meaning of life, expressed the extent to which one has a pervasive, enduring though dynamic feeling of confidence. Antonovsky believed that sense of coherence alleviated stress through three mechanisms of affecting stress assessment, enhancing coping ability and enhancing emotional regulation ability, so it can alleviate social anxiety [13]. Sense of coherence and its three dimensions (sense of comprehensibility, sense of control and sense of meaning) are significantly negatively correlated with social anxiety, individuals with a stronger sense of coherence have a lower level of social anxiety [14, 15]. It is not difficult to found that both of perceived social support and sense of coherence can negatively predict social anxiety. In addition, perceived social support can promote sense of coherence, it has been found that the higher the social support, the stronger the individual's sense of coherence in adolescents and college students, [16-18].

Existing studies have found that enhancing perceived social support can reduce social anxiety, and enhancing coherence can also reduce social anxiety, but there is no research on the mechanism of action of the three. This study attempts to investigate the mediating factors of the perceived consistency between social support and social anxiety. In addition, a recent study showed that childhood major adverse events increased the risk of some social anxiety symptoms, especially experiencing 3 or more adverse events. A sense of security mediated the relationship between childhood major adverse events and social anxiety symptoms [19]. It demonstrates that the individual experiencing more adverse events will induce social anxiety, which increases the significance of the case study experience. In contrast, this study starts from the perspective of positive psychology, the social support perceived by the individual has the meaning of positive happiness. Perceived social support as an external resource for a sense of coherence increases the perceived social support while enhancing the sense of coherence. Individuals are more stable and have a stronger ability to withstand stress, buffering their anxiety, may reduce social anxiety in interacting with others, and provide a theoretical basis for college students to use self-help procedures to prevent and reduce social anxiety, which is of practical significance. This study did not fully consider the role of the quality and stability of various external social support perceived by college students in this mechanism, and it is worthy of further exploration in the following.

\section{Methods}

\subsection{Subjects}

A total of 420 students from eight universities in Shandong and Yunnan provinces were selected by convenience sampling. 420 questionnaires were sent out and 420 questionnaires were returned. Among the respondents, 169 male (42.23\%) and 251 female (59.76\%); freshmen 87 (20.71\%), sophomores 88 (20.95\%), juniors 141 (33.57\%), and senior104 (24.76\%) are seniors, born in rural 340(80.95\%), born in city 80(19.05\%). 


\subsection{Measures}

\subsubsection{Perceived Social Support (independent variable)}

The perceived social support scale (PSSS) was used to measure perceived social support of college students, which selected from the handbook of Mental Health Rating Scale (Revised Version) [20]. "Leaders, relatives, colleagues" was changed to "teachers, classmates, relatives". The scale has 12 items, which is a 7-point scale, with the options ranging from 1 = strongly disagree to 7 = strongly agree. The higher the score, the higher the degree of perceived social support. In this study, the Cronbach's $\alpha$ was 0.94 , indicating high reliability.

\subsubsection{Sense of Coherence (Mediation)}

The Sense of Coherence (SOC-13) was used to measure sense of coherence of college students, which comes from the simplified version of life orientation questionnaire compiled by Antonovsky (1987), and revised by L.P. Bao et al to suit Chinese background [21]. The scale has 13 items, which is a 7-point scale, and the scoring criteria were different according to the different contents of the items. Items 1,2,3,5 and 13 were reverse scoring. The higher the score was, the higher the sense of coherence level was. In this study, the Cronbach's $\alpha$ was 0.77 , indicating good reliability.

\subsubsection{Social anxiety}

The Interaction Anxiousness Scale (IAS) was used to measure social anxiety of college students, which also selected from the handbook of Mental Health Rating Scale (Revised Edition). The scale has 15 items, which is a 5-point scale, with the options ranging from $1=$ "not at all" to $7=$ "very much". In addition, item 3, 6, 10 and 15 are reverse scoring questions. The higher the total score, the higher the level of social anxiety. In this study, the Cronbach $\alpha$ coefficient of the scale was 0.86 , indicating good reliability.

\subsection{Analysis}

In this study, SPSS 21.0 and Mplus7 were used for data collation and analysis. SPSS 21 was used to test the differences in demographic variables of social anxiety and Pearson's correlation test among social anxiety, perceived social support and sense of coherence. The mediating model was validated using Mplus7, in which 95\% confidence intervals (CI) were used and 2000 bootstrapping resamples were run.

\section{Results}

\subsection{Social Anxiety of College Students}

Taking gender, place of birth and grade as independent variables respectively and social anxiety as dependent variable, the independent sample $t$-test or analysis of ANOVA were conducted, and the results showed that social anxiety of female is significantly higher than male, $t(418)=18.99, p<0.001$, there is no significant difference in in grade and place of birth.

Table 1. Social anxiety of different types of college Students $(\mathrm{N}=420)$ 


\begin{tabular}{|c|c|c|c|c|c|}
\hline \multicolumn{2}{|c|}{ type } & $\mathbf{N}$ & $\mathrm{M} \pm \mathrm{SD}$ & $F / t$ & $\mathbf{p}$ \\
\hline \multirow{2}{*}{ gender } & male & 169 & $42.23 \pm 6.08$ & 18.99 & $0.00 * *$ \\
\hline & female & 251 & $45.049 \pm 6.75$ & & \\
\hline & & & & & \\
\hline \multirow{4}{*}{ grade } & freshman & 87 & $43.17 \pm 6.26$ & 2.27 & 0.08 \\
\hline & sophomore & 88 & $42.82 \pm 6.26$ & & \\
\hline & junior & 141 & $44.23 \pm 6.82$ & & \\
\hline & senior & 104 & $45.03 \pm 6.96$ & & \\
\hline & & & & & \\
\hline \multirow{2}{*}{ birthplace } & rural & 340 & $44.01 \pm 6.66$ & 0.36 & 0.55 \\
\hline & city & 80 & $43.51 \pm 6.51$ & & \\
\hline
\end{tabular}

Note: $* * p<0.0$

\subsection{The relationship among social anxiety, perceived social support and sense of coherence}

\subsubsection{The correlation relationship}

\begin{tabular}{|c|c|c|c|}
\hline Variable & $\begin{array}{c}\text { Perceived Social } \\
\text { Support }\end{array}$ & sense of coherence & social anxiety \\
\hline Perceived Social Support & 1 & & \\
\hline sense of coherence & $-0.27^{* *}$ & 1 & \\
\hline social anxiety & $-0.24^{* *}$ & $0.41^{* *}$ & 1 \\
\hline
\end{tabular}

The correlation analysis showed that a significant negative correlation between perceived social support and social anxiety $(r=-0.27, p<0.01)$, sense of coherence was also significantly negative correlation with social anxiety $(r=-0.24, p<0.01)$, and perceived social support was significantly positive correlation with sense of coherence $(\mathbf{r}=0.41, p<$ $0.01)$.

\subsubsection{The mediation models.}

Based on the results of correlation analysis, the mediating effect test was conducted according to the corresponding procedure proposed by Z.L. Wen et al. [19]. Three equations, $\mathrm{Y}=c \mathrm{X}$ (Equation 1), $\mathrm{M}=\mathrm{aX}$ (Equation 2) and $\mathrm{Y}=c^{\prime} \mathrm{X}+b \mathrm{M}$ (Equation 3), are constructed. The independent variable $\mathrm{X}$ is perceived social support, the mediation $\mathrm{M}$ is sense of coherence, and the dependent variable $\mathrm{Y}$ is social anxiety.

The first step to test total effects, social anxiety was associated with lower levels of perceived social support (path $c$ : $\beta=-0.31$, CI [-0.42 -0.19]), indicating the total effect is significant $(p<0.01)$. The second step is to test the regression coefficient $a$ of perceived social support to sense of coherence, and the regression coefficient $b$ of sense of coherence to social anxiety, perceived social support was a positive predictor of sense of coherence, and sense of coherence was a negative predictor of social anxiety (path $a$ : $\beta=0.55$, CI [0.42 0.67], path b: $\beta=-0.14$, CI [-0.24 -0.04]), both path $a$ and $b$ were significant (p $<0.01$ ), indicating significant indirect effect. The third step is to control the sense of 
coherence, and test the direct effect of perceived social support on social anxiety, social anxiety was associated with lower levels of perceived social support (path $c^{\prime}: \beta=-0.24$, CI $[-0.36 \sim-0.11])$, direct effect is also significant $(p<0.01)$. And path $a b, c^{\prime}$ are the same sign, the mediating effect is significant, sense of coherence played a partial mediation in perceived social support and social anxiety, and the mediating effect accounted for $24.83 \%$ of the total effect(ab/c).

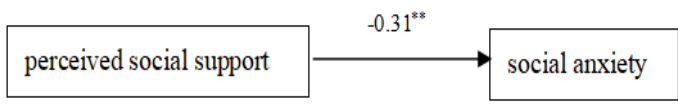

Fig 1 The path map of perceived ssocial support predicting college students' social anxiety

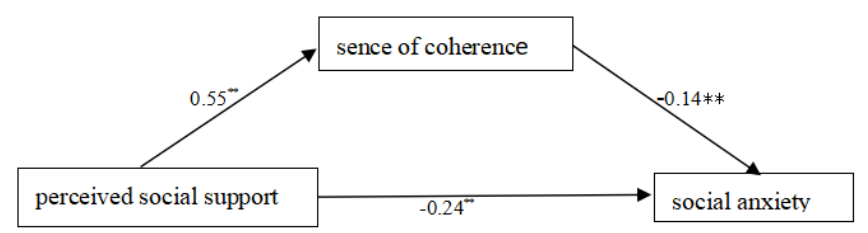

Fig 2 The mediating model of sense of coherence between perceived social support and social anxiety

\section{Discussions}

\subsection{Characteristics of college students' social anxiety}

According to the Manual of Mental Health Rating Scale, C.Z. Peng revised the norm of social anxiety of Chinese college students as (38.78 \pm 8.58$)$, the score of social anxiety of college students this study $(43.88 \pm 6.33)$ is also higher than norm of social anxiety. The follow-up studies from 1998 to 2015, the degree of social anxiety in college students has been increasing [22], the results of this study also accord with this trend. With the development of science and technology and the popularization of the network, the society is gradually transiting from human to human and network society. On the contrary, the ability to communicate with people is regressive, and people's social anxiety is widespread. In addition, we found that students groups of female's social anxiety is higher than male, the result is consistent with previous studies[23], because of the social and cultural prejudice, female have lower expectations of themselves, it makes female have inferior idea in interpersonal interaction, often makes them think that "they are not as good as others", it will affect their self-confidence and self-esteem in the long term, lead to higher anxiety level than male in interpersonal communication [24].

\subsection{The correlation relationship among social anxiety, perceived social support and sense of coherence}

Through Pearson correlation analysis, it is found that social anxiety, perceived social support and sense of coherence are significantly correlated in pairs. It is consistent with the results of previous studies, there was a significant positive correlation between perceived social support and sense of coherence, suggests that perceived social support can effectively affect an individual's sense of coherence as an external resource. And sense of coherence and college students' social anxiety has significant negative correlation, the higher sense of 
coherence, the lower social anxiety. Individuals with a high sense of coherence, believe that they can use the beneficial resources to relieve the pressure in the external environment, so that they can maintain their inner stability in social interaction, naturally face to various social situations. It is found that a significant negative correlation between perceived social support and social anxiety, which indicates that the higher the perceived social support is, the improved social anxiety will be to some extent. When individuals can better understand the support come from family, friends and others, they will have more confidence in their own social level and ability, and feel more goodwill from the people around them. Therefore, they can trust others in social situations, thus reducing the level of social anxiety.

\subsection{Sense of coherence mediated perceived social support and social anxiety}

This study found that sense of coherence played a partial mediation in perceived social support and social anxiety. Perceived social support has a direct effect on social anxiety of college students, and also plays an in director through the sense of coherence. It has some practical significance that sense of coherence' mediating effect between perceived social support and social anxiety. On the one hand, we found that perceived social support was not only directly related to social anxiety, but also indirectly related to social anxiety through sense of coherence. On the other hand, this finding is conducive to the intervention of college students' interpersonal relationship. Before the occurrence of interpersonal relationship obstacle, it can strengthen their sense of coherence, which is conducive to relieving the tension in the social situation. All in all, this finding is of great significance not only to enrich the theory of this field, but also to carry out the mental health work of college students.

\section{Conclusions}

Through this questionnaire survey, we found that college students have a higher degree of social anxiety. And there is a positive correlation between perceived social support and sense of coherence, and a negative correlation between social anxiety and both perceived social support and sense of coherence. Furthermore, sense of coherence plays a partial mediation in between perceived social support and social anxiety. This study provides theoretical support for revealing the mechanism between perceived social support and social anxiety.

\section{References}

1. X.W. Guo, Psychological Exploration, Research on the Causes of Social Anxiety in College Students, 20(1), 55-58, 2000

2. M. Shi, N. Li, W.Y. Lu, X.Y. Yu \& S.F. Xin, Psychological Research, A crosssectional historical study on the changes of social anxiety among Chinese college students, 012(006), 540-547, 2019

3. B. Li, J. Zhong, M.Y. Qian, Chinese Journal of Mental Health, Regression analysis of susceptibility of college students to social anxiety, (002), 108-112, 2003

4. S.Y. Ren, Journal of Yangzhou University, Investigation and analysis of college students' social anxiety, (06), 75-78, 2007

5. C.Y. Moutier, M.B. Stein, The Journal of Clinical Psychiatry, The history, epidemiology, and differential diagnosis of social anxiety disorder, 60(Supply 9), 4-8, 1999 
6. Jefferiesid, P., Ungar, M. Social anxiety in young people: A prevalence study in seven countries. Plos One 15, e0239133,2020

7. S.Y. Xiao, D.S. Yang, Chinese Journal of Mental Health, The impact of social support on physical and mental health, (04), 183-187, 1987

8. Q. Zhang, R.G. Wu, S.W. Hao, Z.L. Xu, \& R.Y. Guan, Chinese Journal of Health Psychology. The Mediating Role of Self-esteem in perceived Social Support and Depression among College Students, (25), 89-93, 2017

9. H.X. Xu, Chinese Journal of Health Psychology, The influence of perceived social support on college students' depression, 04, 415-417, 2008

10. E. Reinelt, European Archives of Psychiatry and Clinical Neuroscience, High social support buffers the effects of 5-HTTLPR genotypes within social anxiety disorder. 264(5), 2014

11. X. Dong, Y. Zeng, Higher education BBS, A study on the relationship between social anxiety and social support among undergraduates, (11), 54-57, 2020

12. M.D. Barnett, L.V. Maciel, D.M. Johnson, \& I. Ciepluch, Psychological Reports, Social anxiety and perceived social support: gender differences and the mediating role of communication styles, 124(1), 003329411990097. 2020

13. A. Antonovsky, Social Science \& Medicine, The structure and properties of the sense of coherence scale. 36(6), 725, 1993

14. T.T Zhan, Anhui Medical University, A study on the relationship between sense of coherence, social support, coping style and subjective well-being of nurses, 2009

15. Y. Wang, X.J.Jin, L.J. Ren, Chinese Journal of Health Psychology, The correlation between sense of coherence, social anxiety and temperament types of college students, 22(001), 140-142, 2014

16. S.C. Marsh, S.S. Clinkinbeard, R.M. Thomas, Journal of Health Psychology, Risk and Protective Factors Predictive of Sense of Coherence during Adolescence, 12(2), 281284, 2007

17. Y.W. Ying, P.A. Lee, J.L. Tsai, American Journal of Orthopsychiatry, Predictors of depressive symptoms in Chinese American college students: parent and peer attachment, college challenges and sense of coherence, 77(2), 316-323, 2010

18. H. Wan, X.Y. Wang, The Writer of Heaven and Earth. The Relationship between Social Support and Positive Coping among College Students: The Mediating Role of sense of coherence. 07, 86-87, 2020

19. Tian-tian Meng, etc. Journal of Affective Disorders, Analysis of features of social anxiety and exploring the relationship between childhood major adverse experiences and social anxiety in early adulthood among Chinese college students, (06),2021

20. X.D. Wang, X.L. Wang, L. Ma, Chinese Journal of Mental Health, Manual of Mental Health Rating Scales (Revised Version), 131-133, 230-232, 1999

21. L.P. Bao, J.S. Liu, Y. Zhou, Chinese Journal of Mental Health, A Preliminary Study on Reliability and Validity of sense of coherence (SOC-13), (05), 299, 2006

22. Z.L. Wen, B.J. Ye, Advances in Psychological Science, Mediating effect analysis: methodology and model development, (005), 731-745, 2014

23. Q.Y. Wang, Journal of Liaoning University of Science and Technology, the status quo and thinking of college students' social anxiety, 020(003), 102-104, 2018

24. B.W. Luo, Psychological Science, A preliminary study on the causes of female high anxiety tendency, (04), 378-379, 1997 